\title{
Effects of Head Positions on Awake Orotracheal Intubation Via Fiberoptic-Bronchoscope: A Randomized Controlled Trial
}

\section{Zhuo Liu ( $\sim$ liuzhuo2011@yeah.net)}

The first hospital of Qinhuangdao https://orcid.org/0000-0003-2449-9697

\section{Li Zhao}

First Hospital of Qinhuangdao

Meiqi Liu

First Hospital of Qinhuangdao

\section{Xiaohang Qi}

First Hospital of Qinhuangdao

\section{Qianqian Jia}

First Hospital of Qinhuangdao

\section{Shujuan Liang}

First Hospital of Qinhuangdao

\section{Xiaochun Yang}

First Hospital of Qinhuangdao

\section{Research article}

Keywords: Awake orotracheal intubation, fiberoptic-bronchoscope, head positions

Posted Date: November 10th, 2020

DOl: https://doi.org/10.21203/rs.3.rs-102669/v1

License: (c) (i) This work is licensed under a Creative Commons Attribution 4.0 International License. Read Full License 


\section{Abstract}

Background: There are many factors affect the success rate of awake orotracheal intubation via fiberoptic bronchoscope. We performed this study to investigate the effects and safety of three head positions on awake orotracheal intubation via fiberoptic bronchoscope.

Methods: Seventy-five adult patients with anticipated difficult airway, received general anaesthesia and undergoing awake orotracheal intubation were included in this study. According to the head position, the patients were randomized allocated to neutral position group (NP group), sniffing position group (SP group) or extension position group (EP group). After conscious sedation the patients were intubated by an experienced anesthesiologist. The time to view the vocal cords, the time to insert the tracheal tube into the trachea, the percentage of glottic opening scores (POGO) and the visual analog scale (VAS) scores for ease experienced of passing the tracheal tube through the glottis, the hemodynamic changes during intubation and the adverse events after surgery were recorded.

Results: The time to view the vocal cords was significantly shorter and the POGO scores was significantly higher in the EP group compared with the other two groups $(\mathrm{P}<0.05)$; the $\mathrm{SpO}_{2}$ immediate after intubation was higher in the EP group compared with SP group $(\mathrm{P}<0.05)$ and the $\mathrm{SpO}_{2}$ before intubation was higher in the EP group compared with NP group $(\mathrm{P}<0.05)$, while at other time points the $\mathrm{SpO}_{2}$ had no significant difference among groups. The time to tracheal intubation, the VAS scores for passing the tracheal tube through glottis, the coughing scores of patients when inserted fiberoptic bronchoscope into the trachea and inserted the tracheal tube into the trachea over fiberoptic bronchoscope had no significant differences among groups $(P>0.05)$. There were also no significant differences among the groups with regard to the incidence of postoperative complications, mean arterial pressure and heart rate at each time point $(P>0.05)$.

Conclusions: Extension position had a best view of glottic opening than neutral position or sniffing position during awake orotracheal intubation via fiberoptic bronchoscope, so extension position was recommended as the starting head position for awake orotracheal intubation using fiberoptic bronchoscope.

Trial registration: Clinical Trials.gov. no. NCT 02792855. Registered at https://register.clinicaltrials.gov on 23 september 2017.

\section{Background}

The incidence of difficult airway is ranging from $0.3-13 \%$ [1] and nearly $30 \%$ of all anaesthesia-related deaths attribute to difficult airway [2]. Awake tracheal intubation via Fiberoptic bronchoscope (FOB) is regarded as the golden standard for the management of difficult airway [3-6]. However, in sedated state patients, the base of tongue, soft palate and epiglottis move backward and obstruct the advancement of FOB $[7,8]$. Recently, several studies have examined the effects of different methods such as jaw thrust, lingual traction or head tilt on FOB intubation [9-15]. However, there have been no studies to determine 
whether the neutral head position, sniffing head position or extension head position is more suitable for Awake Fibreoptic bronchoscope Orotracheal Intubation (AFOI). Thus, the objective of this study was to investigate the effects and safety issues of three head positions during AFOI.

\section{Methods}

This trial was approved by the institutional review board of the first hospital of Qinhuangdao and all patients provided written informed consent.

Seventy-five adult patients with anticipated difficult airway, ASA class I-II, modified Mallampati classification $\geq 3$, requiring general anaesthesia and undergoing awake orotracheal intubation were included in this study. Exclusion criteria were as follows: age $>70$ or $<18$ years, with cervical vertebra disease, incisors loose or missing, preoperative hoarseness, bronchial asthma, a history of airway hyperresponsiveness, hypertension and abnormalities of heart, brain, liver, lung, kidney and coagulation functions.

All these patients were screened by a senior anaesthesiologist preoperatively and according to the head position, the patients were randomized allocated to neutral position group (NP group, Figure A), sniffing position group (SP group, Figure B) or extension position group (EP group, Figure C). Randomization (1:1) was based on computer-generated codes which were kept in sequentially numbered opaque envelopes until the end of study.

All the patients without premedication and received standard monitoring systems, including electrocardiogram (ECG), heart rate (HR), invasive arterial blood pressure (NIBP) and peripheral oxygen saturation $\left(\mathrm{SpO}_{2}\right)$ in the operating room. The patients were intravenously injected with midazolam 0.03 $\mathrm{mg} \cdot \mathrm{kg}^{-1}$. After mild sedation, a intratracheal injection was performed at the cricothyroid membrane with a fine needle and administered with $2 \%$ lidocaine $(3-4 \mathrm{ml})$, then the patients were suggested to open the mouth as wide as possible and then the oral cavity and hypopharynx mucosa were sprayed with $2 \%$ lidocaine. After intratracheal anaesthesia, dexmedetomidine was administered at a loading dose of $1 \mu \mathrm{g} \cdot \mathrm{kg}^{-1}$ (the infusion was completed in 10 minutes) then remifentanil was given at a loading dose of $0.5 \mu \mathrm{g} \cdot \mathrm{kg}^{-1}$, followed by a continuous infusion at a speed of $0.1-0.15 \mu \mathrm{g} \cdot \mathrm{kg}^{-1} \cdot \mathrm{min}^{-1}$. During this process the patients received continuous oxygen by mask at a rate of $5 \mathrm{~L} / \mathrm{min}$.

Before intubation, a FOB (external diameter $5.2 \mathrm{~mm}$, MDHAO Medical Technology Co, Ltd, Zhuhai china) was loaded with a tracheal tube (inner diameter $6.5 \mathrm{~mm}$ for female and inner diameter $7.0 \mathrm{~mm}$ for male) and lubricated with dyclonine hydrochloride mucilage. After deep sedation (patients breathing spontaneously but cannot be awakened by call her name), the intubation was performed and the steps were as follows: First, a bite block was placed between the teeth of patients then the operator inserted the FOB into the mouth with his left thumb and forefinger, simultaneously gripped the chin upward with the rest of his fingers. At this point, the bite block as a fulcrum to lift the mandible upward to open the space of pharynx and laryngeal cavity as far as possible (Figure A, B, C) then advanced the FOB downward 
along the oropharyngeal curve with his left thumb and forefinger until viewing the epiglottis and glottis. After the anterior of FOB passing the vocal cords, the FOB was inserted into the trachea and then the tracheal tube was pushed into the trachea over the FOB. Orotracheal intubation was suspended when $\mathrm{SpO}_{2}<90 \%$ and oxygen was supplied by mask.

The primary outcome parameters of the study were the time to view the vocal cords (TVVC) and the percentage of glottic opening scores (POGO). TVVC was defined as the time from inserting the FOB between the teeth until the operator view the the entire vocal cords; POGO was the percentage of glottic opening scores (POGO) to evaluate the operator's first vision of glottic opening during the FOB just passed the tongue base (score range:0-100: $0=$ none, $100=$ full) $(11,16)$. The time to advance the tracheal tube into trachea over FOB (TATT) defined as the time from the operator viewing the entire vocal cords to insert the tracheal tube into trachea successfully, the coughing scores when inserted the FOB into trachea and inserted the tracheal tube into trachea via FOB (coughing score: $0=$ none, $1=$ slight, $2=$ moderate, $3=$ severe) (17), the VAS scores of the ease experienced for inserting the tracheal tube into trachea indicated by the operator immediately after the intubation (score range:0-100, $0=$ very difficult, $100=$ very easy), the hemodynamic alterations of patients during intubation and postoperative complications such as hoarseness and pain of throat were also recorded. TVVC and TATT were assessed by an independent observer with a stopwatch.

In our study, the sample size was determined according to a pilot study which we recorded the POGO scores and measured the time to view the vocal cords. With significance set at 0.05 and power set at $80 \%$, the sample size required to detect differences was 20 patients each group. Thus, we recruited 25 patients each group to prevent unforeseen difficulties.

Analyses were performed using SPSS 21.0 statistical software. Continuous variables were presented as mean \pm standard deviation (SD) and the differences among the groups were compared with ANOVA (Bonferroni or Dunnett T3 test was used for multiple comparisons). The differences of proportions were analyzed using Kruskal-Wallis test. The differences of the incidence were analyzed with Fisher's exact test. $P$ value less than 0.05 was considered as statistically significant.

\section{Results}

All patients were completed the AFOI. There were no significant differences in the demographic characteristics of patients among groups (Table 1). 
Table 1

Demographic characteristics of patients

\begin{tabular}{|c|c|c|c|c|}
\hline Characteristics of patients & $S P(n=25)$ & $N P(n=25)$ & $\operatorname{EP}(n=25)$ & $P$-value \\
\hline Age (years) & $50.8 \pm 13.2$ & $51.0 \pm 11.1$ & $53.9 \pm 10.3$ & 0.58 \\
\hline Gender $(M / F, n)$ & $11 / 14$ & $14 / 11$ & $13 / 12$ & 0.68 \\
\hline ASA $(I / I I, n)$ & $15 / 10$ & $12 / 13$ & $14 / 11$ & 0.32 \\
\hline Weight (kg) & $68.9 \pm 16.2$ & $70.7 \pm 9.9$ & $70.5 \pm 11.9$ & 0.87 \\
\hline Height (cm) & $165.1 \pm 9.0$ & $166.9 \pm 6.8$ & $167.4 \pm 7.8$ & 0.58 \\
\hline $\mathrm{BMI}\left(\mathrm{kg} / \mathrm{m}^{2}\right)$ & $25.1 \pm 4.2$ & $25.4 \pm 3.3$ & $25.1 \pm 3.4$ & 0.95 \\
\hline Thyromental distance $(\mathrm{cm})$ & $5.1 \pm 0.6$ & $5.5 \pm 0.5$ & $5.2 \pm 0.9$ & 0.26 \\
\hline Mouth opening $(\mathrm{cm})$ & $4.5 \pm 0.9$ & $4.4 \pm 1.5$ & $4.2 \pm 1.1$ & 0.73 \\
\hline $\begin{array}{l}\text { Mallampati class } \\
(3 / 4, n)\end{array}$ & $12 / 13$ & $9 / 16$ & $11 / 14$ & 0.68 \\
\hline Degree of neck extension & & & & 0.49 \\
\hline$>90^{\circ}(\mathrm{n})$ & 21 & 24 & 23 & \\
\hline$\leq 90^{\circ}(\mathrm{n})$ & 4 & 1 & 2 & \\
\hline
\end{tabular}

The time to view the vocal cords was significantly shorter and the POGO scores was significantly higher in the EP group compared with the other two groups $(\mathrm{P}<0.05)$; the $\mathrm{SpO}_{2}$ immediate after intubation was higher in the EP group compared with SP group $(\mathrm{P}<0.05)$ and the $\mathrm{SpO}_{2}$ before intubation was higher in the EP group compared with NP group $(\mathrm{P}<0.05)$, while at other time points the $\mathrm{SpO}_{2}$ had no significant difference among groups. The time to tracheal intubation, the VAS scores for passing the tracheal tube through glottis, the incidence of postoperative complications, the coughing scores of patients when inserted $\mathrm{FOB}$ into the trachea and inserted the tracheal tube into the trachea over FOB had no significant differences among groups $(P>0.05)$ (Table 2$)$. 
Table 2

Comparison of the time to view the vocal cords, time to advance tracheal tube into trachea, percentage of glottic opening scores, the VAS scores for ease of passing the tracheal tube through the glottis, the

coughing scores and the adverse events after tracheal intubation.

\begin{tabular}{|c|c|c|c|c|}
\hline Factor & $\begin{array}{l}\text { SP } \\
25)\end{array}$ & $\operatorname{NP}_{25)}(n=$ & $\operatorname{EP}(n=25)$ & $\begin{array}{l}P \\
\text { value }\end{array}$ \\
\hline Time to view the vocal cords(s) & $\begin{array}{l}20.1 \pm \\
9.9\end{array}$ & $\begin{array}{l}22.5 \pm \\
10.5\end{array}$ & $\begin{array}{l}14.2 \pm \\
4.9^{\star \#}\end{array}$ & $<0.01$ \\
\hline $\begin{array}{l}\text { Time to advance tracheal } \\
\text { tube into trachea (s) }\end{array}$ & $\begin{array}{l}17.4 \pm \\
5.8\end{array}$ & $19.8 \pm 9.6$ & $17.5 \pm 8.1$ & 0.51 \\
\hline $\begin{array}{l}\text { Percentage of glottic } \\
\text { opening scores }\end{array}$ & $\begin{array}{l}78.5 \pm \\
20.5\end{array}$ & $\begin{array}{l}75.2 \pm \\
19.6\end{array}$ & $\begin{array}{l}88.9 \pm \\
11.5^{\star \#}\end{array}$ & 0.02 \\
\hline $\begin{array}{l}\text { VAS scores for tracheal } \\
\text { tube passing glottis }\end{array}$ & $\begin{array}{l}87.4 \pm \\
13.7\end{array}$ & $\begin{array}{l}79.3 \pm \\
23.5\end{array}$ & $82.4 \pm 19.4$ & 0.33 \\
\hline Coughing scores of inserting FOB $(0 / 1 / 2 / 3, n)$ & $23 / 2 / 0 / 0$ & $22 / 2 / 1 / 0$ & $24 / 1 / 0 / 0$ & 0.94 \\
\hline $\begin{array}{l}\text { Coughing scores of inserting tracheal tube }(0 / 1 \\
/ 2 / 3, n)\end{array}$ & $12 / 5 / 7 / 1$ & $11 / 7 / 4 / 3$ & $14 / 7 / 2 / 2$ & 0.98 \\
\hline Throat pain(n) & 6 & 6 & 8 & 0.76 \\
\hline Hoarseness (n) & 1 & 2 & 1 & 0.77 \\
\hline
\end{tabular}

There were also no significant differences among the groups with regard to mean arterial pressure and heart rate during intubation $(P>0.05)$ (Table 3$)$. 
Table 3

Hemodynamic alterations of patients during intubation.

\begin{tabular}{|c|c|c|c|c|}
\hline Factor & $S P(n=25)$ & $N P(n=25)$ & $\operatorname{EP}(n=25)$ & $P$-value \\
\hline \multicolumn{5}{|c|}{ MAP $(\mathrm{mmHg})$} \\
\hline TO & $96.3 \pm 10.2$ & $101.2 \pm 9.5$ & $100.0 \pm 11.1$ & 0.22 \\
\hline T1 & $91.1 \pm 13.5$ & $92.3 \pm 12.2$ & $97.4 \pm 11.2$ & 0.18 \\
\hline T2 & $97.8 \pm 14.8$ & $100.0 \pm 14.6$ & $105.8 \pm 13.4$ & 0.13 \\
\hline T3 & $91.3 \pm 14.8$ & $95.7 \pm 13.4$ & $98.5 \pm 13.5$ & 0.18 \\
\hline $\mathrm{T} 4$ & $87.5 \pm 13.5$ & $90.2 \pm 14.3$ & $94.4 \pm 11.9$ & 0.21 \\
\hline \multicolumn{5}{|l|}{ HR (bpm) } \\
\hline TO & $74.1 \pm 13.4$ & $72.8 \pm 13.4$ & $75.7 \pm 8.7$ & 0.71 \\
\hline T1 & $67.3 \pm 12.8$ & $64.8 \pm 10.9$ & $66.3 \pm 10.8$ & 0.74 \\
\hline T2 & $71.2 \pm 15.1$ & $64.6 \pm 10.6$ & $64.4 \pm 11.6$ & 0.11 \\
\hline T3 & $65.6 \pm 11.3$ & $61.7 \pm 10.0$ & $62.4 \pm 11.7$ & 0.40 \\
\hline T4 & $65.4 \pm 12.4$ & $60.9 \pm 8.4$ & $62.2 \pm 11.0$ & 0.33 \\
\hline $\mathrm{SpO}_{2}(\%)$ & & - & & \\
\hline TO & $97.5 \pm 1.6$ & $98.1 \pm 1.8$ & $98.5 \pm 1.5$ & 0.10 \\
\hline T1 & $97.7 \pm 2.4$ & $96.4 \pm 3.5$ & $98.8 \pm 2.0^{\#}$ & 0.01 \\
\hline T2 & $96.4 \pm 5.1$ & $97.6 \pm 2.2$ & $99.1 \pm 1.4^{\star \#}$ & 0.02 \\
\hline T3 & $98.8 \pm 2.1$ & $98.8 \pm 2.4$ & $99.6 \pm 0.6$ & 0.20 \\
\hline $\mathrm{T} 4$ & $99.5 \pm 0.7$ & $99.3 \pm 1.4$ & $99.2 \pm 1.5$ & 0.73 \\
\hline \multicolumn{5}{|c|}{$\begin{array}{l}\text { MAP, mean arterial pressure; HR, heart rate; } \mathrm{SpO}_{2} \text {, pulse oxygen saturation. T0, Before anesthesia; } \mathrm{T} 1 \\
\text { Before intubation; T2, Immediate after intubation; T3, } 1 \text { min after intubation; } \mathrm{T} 4,3 \mathrm{~min} \text { after } \\
\text { intubation. }{ }^{\star} P<0.05 \text { compared with SP group, }{ }^{\sharp} P<0.05 \text { compared with NP group. }\end{array}$} \\
\hline
\end{tabular}

\section{Discussion}

Inability to maintain patient's airway associate with hypoventilation, hypoxemia, hypoxic brain injury or even death [18-20], so the management of the difficult airway is at high-risk. Awake intubation via FOB is still the recommended method for dealing with difficult airway [3-6] and a clear airway that allows the passage of the FOB is critical to awake intubation via FOB. 
In this study, we compared the effect and safety issues of three head positions (neutral position, sniffing position and extension position) during AFOI. The results showed us that the time for viewing the vocal cords was significantly shorter and the POGO scores were significantly higher in the extension position group compared with the other two groups, in addition at before intubation, the $\mathrm{SpO}_{2}$ was higher in the EP group compared with neutral position group and at immediate after intubation the $\mathrm{SpO}_{2}$ was also higher in the EP group compared with the sniffing position group. The results suggested that the extension position facilitated the FOB passing through the base of the tongue and easy to view the whole glottis. The results of $\mathrm{SpO}_{2}$ also confirmed that the extension position was better than sniffing position or neutral position to keep the airway open in sedated patients.

Tracheal intubation via laryngoscope requires the operator to view the glottis directly and clearly, therefore, how to reduce the angle of airway axes (oral, pharyngeal and laryngeal) is the key to successful intubation. Magill first reported that the sniffing position was better than the neutral position during tracheal intubation via laryngoscopy [21]. This recommendation is based on the so-called three-axes alignment theory that the sniffing position make three axes closer alignment [22, 23]. Although the clinical benefit of sniffing position is controversial $[24,25]$, sniffing position is still recommended for tracheal intubation using laryngoscopy [26, 27].

It seems to be different of tracheal intubation using FOB for the anterior of FOB can move freely, so the FOB can cross the angle among the three axes, however the FOB can not provide enough support to the laryngeal tissue, resulting in the base of tongue, soft palate and epiglottis were closer to the posterior pharyngeal wall [28], lead to the operater unable to find the glottis easily in sedated patients using FOB. Therefore, sufficient space of pharyngeal cavity and laryngeal cavity is necessary for FOB to successfully pass through the base of the tongue and quickly view the glottis during AFOI. So the space of pharyngeal cavity and laryngeal cavity is the key to successful AFOI. Several studies found that the manoeuvre of jaw-thrust elevated the epiglottis and tongue base away from the wall of posterior pharyngeal, provided more space in the pharyngeal cavity and laryngeal cavity [9-14]. So the manoeuvre of jaw thrust facilitated viewing the vocal cords and increased the success rate of oral FOB intubation [29]. Durga et al demonstrated that used jaw thrust and lingual traction alone unable to clear the airway completely, whereas the combining used of jaw thrust and lingual traction cleared the airway more effectively [10]. Stacey et al found a clear airway at the level of the palate and larynx with a combined of FOB and laryngoscopy technique [11]. The problem with these methods is that one or two trained assistants are required. Therefore, in some emergency situations, such as unpredictable difficult airway the success rate of orotracheal intubation via FOB may reduce for without the aid of assistants. In addition, during awake orotracheal intubation the patients only sedated and without the use of muscle relaxants, so the manoeuvre of jaw thrust, lingual traction or assistance by laryngoscope may increase the discomfort of patients and cause the resistance of patients. In present study, all patients in the SP group with head and cervical extension by placing a 7-cm firm pillow under the shoulders of patients, so this positions tightened the muscles and tissues in the front of neck then moved the oropharyngeal structures anteriorly and emptied the oropharyngeal airspace. In addition, with head extension position, the operator made the 
bite block as a fulcrum and lifted the mandible upward more easily than other two positions during intubation, which may achieve similar effects as jaw thrust that lifting the base of tongue enabling the FOB easily to pass through the base of the tongue. Most important one was the whole process of intubation with no need for the assistance of others.

Successful inserting a FOB into the trachea does not guarantee a successful AFOI, because the anterior of the tracheal tube may impinge on the laryngeal structures when advance the tracheal tube into trachea over the FOB [30,31], which may lead to serious injury of laryngeal [32] or be catastrophic in some cases [14]. This difficulty can be reduced by reducing the gap between the tracheal tube and FOB [33]. In this study, the outside diameter of FOB was $5.2 \mathrm{~mm}$ and the inner diameter of tracheal tube was $6.5 \mathrm{~mm}$ for female and $7.0 \mathrm{~mm}$ for male, so the gap between the tracheal tube and FOB was very small, furthermore we used silicone flexible tracheal tube and the inside and outside of the tracheal tube was lubricated with dyclonine hydrochloride mucilage, so the advancement of a tracheal tube over the FOB very smoothly in all the patients.

In our study, we found no statistical differences in the hemodynamic changes and coughing scores among groups, the reasons may be that topical anaesthesia of the trachea and combined use of dexmedetomidine and remifentanil not only preserved the patients' spontaneous breathing but also achieve adequate sedation depth during intubation [34].

The patients were interviewed by an independent anesthesiologist one day after operation and the results showed us that all the patients were unable to recall the procedure of topical anaesthesia and intubation, the reasons may be that the application of low dose midazolam and the combined use of dexmedetomidine and remifentanil elimination of the patients' memory of these procedure. In addition, the small size of the tracheal tube was selected and the tracheal tube was adequately lubricated with dyclonine hydrochloride mucilage which may reduced the incidence of adverse complications and improved the comfort of patients.

There were also some limitations in our study. First, this method is not suitable for patients with cervical vertebra sickness; Second, this method is not suitable for patients with incisors loose or missing; Third, this method may not be applicable to the patient with oversize-chin or the operator with little experience in the management of FOB; Fourth, it was not possible to blind investigator to the technique, consequently we cannot rule out the possibility of biases by investigator in this study.

\section{Conclusion}

Extension position had a batter view of glottic opening than neutral position or sniffing position during AFOI, so extension position may be recommended as the initial head position for AFOI.

\section{Abbreviations}

POGO 
Percentage of glottic opening scores; VAS:Visual analog scale scores; ASA:America society of anesthesiology; FOB:Fiberoptic bronchoscope; AFOI:Awake Fibreoptic bronchoscope Orotracheal Intubation; TVVC:Time to view the vocal cords; TATT:Time to advance the tracheal tube into trachea over FOB; SPSS:Statistical package for social sciences

\section{Declarations}

\section{Ethics approval and consent to participate}

This trial was approved by the institutional review board of the first hospital of Qinhuangdao and all patients provided written informed consent.

\section{Consent for publication}

Not applicable.

\section{Availability of data and materials}

The datasets are available from the corresponding author on request.

\section{Competing interests}

The authors declare that they have no competing interests

\section{Funding}

Not applicable.

\section{Authors' contributions}

ZL collected all the patient initial data and drafted the manuscript, LZ, MQL and QQJ completed the anesthesia management. XHQ, SJL helped with manuscript editing, and modifying figures and tables. $\mathrm{XCY}$ contributed to the writing, review and editing of the manuscript. All the authors read and approved the final manuscript.

\section{Acknowledgements}

Not applicable.

\section{References}

1. Petrini F, Accorsi A, Adrario E, et al. Recommendations for airway control and difficult airway management. Minerva Anestesiol. 2005;71:617-57.

2. Schmitt $H$, Buchfelder M, Radespiel-Tröger M, Fahlbusch R. Difficult intubation in acromegalic patients: incidence and predict ability. Anesthesiology. 2000; 93: 110-4. 
3. Rosenstock CV, Thogersen B, Afshari A, Christensen AL, Eriksen C. Awake fiberoptic or awake video laryngoscopic tracheal intubation in patients with anticipated difficult airway management: a randomized clinical trial. Anesthesiology. 2012;116:1210-6.

4. Frova G, Sorbello M. Algorithms for difficult airway management: a review. Minerva Anestesiol. 2009; 75:201-9.

5. Law JA, Broemling N, Cooper RM, et al. Canadian Airway Focus Group. The difficult airway with recommendations for management the anticipated difficult airway. Can J Anaesth. 2013;60:1119-38.

6. Schenk A, Markus CK, Kranke P. Awake fiberoptic intubation - gold standard for the anticipated difficult airway. Anasthesiol Intensivmed Notfallmed Schmerzther. 2014;49:92-9.

7. Nandi P, Charlesworth C, Taylor S, Nunn J, Dore C. Effect of general anaesthesia on the pharynx. $\mathrm{Br} \mathrm{J}$ Anaesth. 1991;66:157-162.

8. Sutthiprapaporn P, Tanimoto K, Ohtsuka M, Nagasaki T, lida Y, Katsumata A. Positional changes of oropharyngeal structures due to gravity in the upright and supine positions. Dentomaxillofac Radiol. 2008; 37:130-5.

9. Iqbal R, Gardner-Thorpe C, Thompson J, Popat MT, Yentis SM, Pandit JJ. A comparison of an anterior jaw lift manoeuvre with the Berman airway for assisting fibreoptic orotracheal intubation. Anaesthesia. 2006;61:1048-52.

10. Durga VK, Millns JP, Smith JE. Manoeuvres used to clear the airway during fibreoptic intubation. $\mathrm{Br} \mathrm{J}$ Anaesth. 2001;87:207-11.

11. Stacey M, Rassam S, Sivasankar R, Hall J, Latto I. A comparison of direct laryngoscopy and jaw thrust to aid fibreoptic intubation. Anaesthesia. 2005; 60:445-8.

12. Aoyama K, Takenaka I, Nagaoka E, Kadoya T. Jaw thrust maneuver for endotracheal intubation using a fiberoptic stylet. Anesth \& Analg. 2000;90:1457-58.

13. Han S, Oh A, Jung C, Park S, Kim J, Nahm F. The effect of the jaw-thrust manoeuvre on the ability to advance a tracheal tube over a bronchoscope during oral fibreoptic intubation. Anaesthesia. 2013;68:472-7.

14. Uzun L, Ugur MB, Altunkaya H, Ozer Y, Ozkocak I, Demirel CB. Effectiveness of the jaw-thrust maneuver in opening the airway: a flexible fiberoptic endoscopic study. ORL J Otorhinolaryngol Relat Spec. 2005;67:39-44.

15. Sanghee Park, Hyung Gon Lee, Jeong II Choi, et al. Comparison of vocal cord view between neutral and sniffing position during orotracheal intubation using fiberoptic bronchoscope: a prospective, randomized cross over study. BMC Anesthesiology. 2019;19:3.

16. Ochroch EA, Hollander JE, Kush S, Shofer FS, Levitan RM. Assessment of laryngeal view: percentage of glottic opening score vs Cormack and Lehane grading. Can J Anaesth. 1999;46:987-90.

17. Brydges G, Atkinson R, Perry MJ, Hurst D, Laqua T, Wiemers J. Awake craniotomy: a practice overview. AANA J. 2012; 80(1): 61-8. 
18. Peterson GN, Domino KB, Caplan RA, Posner KL, Lee LA, Cheney FW. Management of the difficult airway: a closed claims analysis. Anesthesiology. 2005;103:33-9.

19. Hove LD, Steinmetz J, Christoffersen JK, Moller A, Nielsen J, Schmidt H. Analysis of deaths related to anesthesia in the period 1996-2004 from closed claims registered by the Danish patient insurance association. Anesthesiology .2007; 106: 675-80.

20. Rosenstock C, Moller J, Hauberg A. Complaints related to respiratory events in anaesthesia and intensive care medicine from 1994 to 1998 in Denmark. Acta Anaesthesiol Scand. 2001; 45: 53-8.

21. Magill IW. Endotracheal anesthesia. Am J Surg. 1936;34:450-5.

22. Bannister F, Macbeth R. Direct laryngoscopy and tracheal intubation. Lancet. 1944; 244:651-4.

23. Ichiro Takenaka, Kazuyoshi Aoyama, Tamao Iwagaki, Hiroshi Ishimura, Tatsuo Kadoya. The sniffıng position provides greater occipitoatlanto-axial angulation than simple head extension: a radiological study. CAN J ANESTH. 2007;54:129-33.

24. Adnet F, Baillard C, Borron SW, Denantes C, Lefebvre L, Galinski M, et al. Randomized study comparing the "sniffing position" with simple head extension for Laryngoscopic view in elective surgery patients. Anesthesiology. 2001;95:836-41.

25. Common Practice IS. Concepts in anesthesia: time for Reassessment is thesniffing position a "gold standard" for laryngoscopy? Anesthesiology. 2001; 95:825-7.

26. Gal TJ. Airway management. Anaesthesiology. 2005:1617-52.

27. Akihisa Y, Hoshijima H, Maruyama K, Koyama Y, Andoh T. Effects of sniffing position for tracheal intubation: a meta-analysis of randomized controlled trials. Am J Emerg Med. 2015;33:1606-11.

28. Mathru M, Esch O, Lang J, Herbert ME, Chaljub G, Goodacre B, et al. Magnetic resonance imaging of the upper airway. Effects of propofol anesthesia and nasal continuous positive airway pressure in humans. Anesthesiology. 1996;84:273-9.

29. Asai T, Murao K, Johmura S, Shingu K. Effect of cricoid pressure on the ease of fibrescope-aided tracheal intubation. Anaesthesia. 2002; 57: 909-13.

30. Asai T, Shingu K. Difficulty in advancing a tracheal tube over a fibreoptic bronchoscope: incidence, causes and solutions. Br J Anaesth. 2004; 92(6):870-81.

31. Hakala P, Randell T, Valli H. Comparison between tracheal tubes for orotracheal fibreoptic intubation. Br J Anaesth. 1999; 82(1):135-6.

32. McGuire G, el-Beheiry H. Complete upper airway obstruction during awake fibreoptic intubation in patients with unstable cervical spine fractures. Canadian Journal of Anesthesia. 1999; 46:176-8.

33. Marfin AG, Iqbal R, Mihm F, Popat MT, Scott SH, Pandit JJ. Determination of the site of tracheal tube impingement during nasotracheal fibreoptic intubation. Anaesthesia. 2006; 61:646-50.

34. Johnston KD, Rai MR. Conscious sedation for awake fibreoptic intubation: a review of the literature. Canadian Journal of Anesthesia. 2013; 60: 584-99.

\section{Figures}



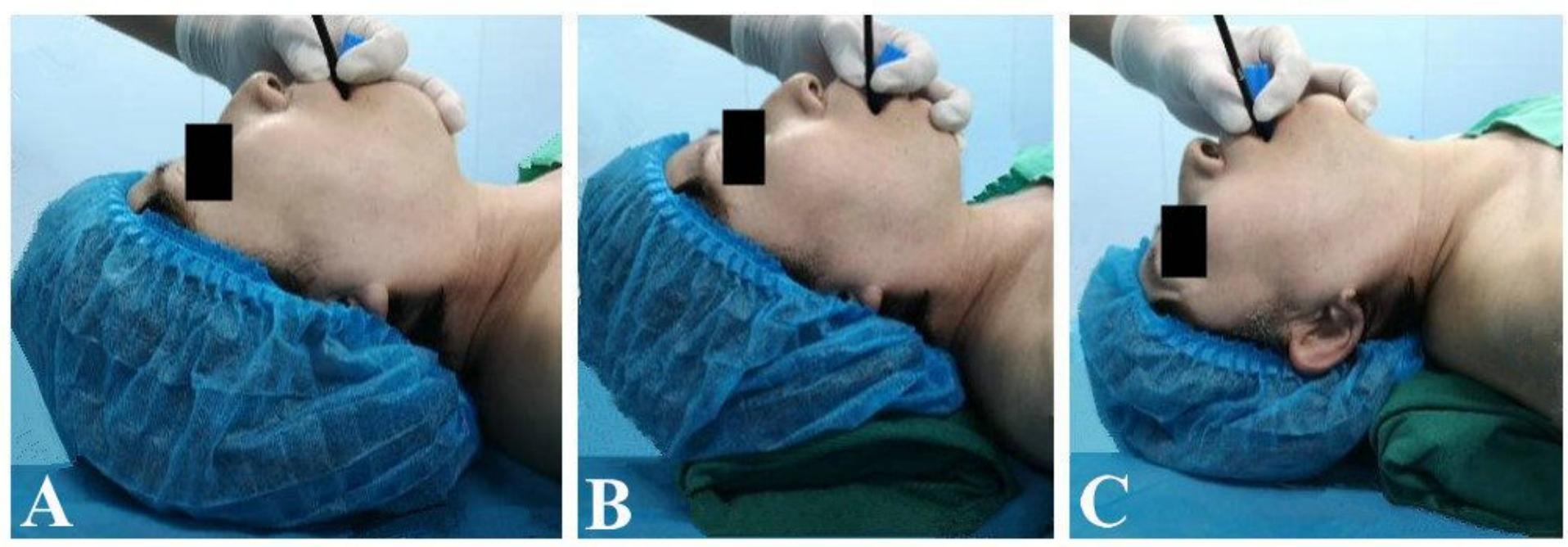

\section{Figure 1}

The position of intubation was demonstrated by a volunteer: Figure A, 'neutral' position with the occiput close to the operating table; Figure B, 'sniffing' position with a 7-cm pillow underneath the occiput; Figure $\mathrm{C}$, 'extension' position with a 7-cm pillow underneath the shoulder and the occiput close to the operating table.
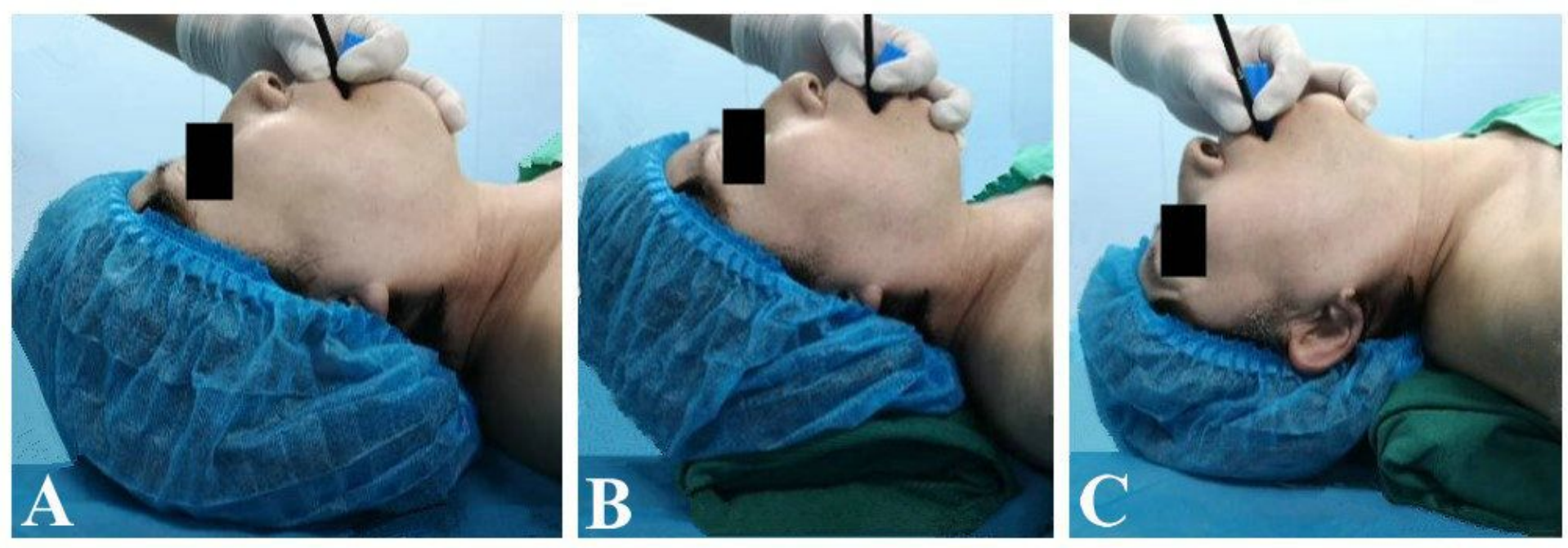

\section{Figure 1}

The position of intubation was demonstrated by a volunteer: Figure A, 'neutral' position with the occiput close to the operating table; Figure B, 'sniffing' position with a 7-cm pillow underneath the occiput; Figure $\mathrm{C}$, 'extension' position with a 7-cm pillow underneath the shoulder and the occiput close to the operating table.

\section{Supplementary Files}

This is a list of supplementary files associated with this preprint. Click to download. 
- CONSORT2010Checklist.doc

- CONSORT2010Checklist.doc 